\title{
KRAS NM_004985.3:c.180_181delTCinsAA
}

National Cancer Institute

\section{Source}

National Cancer Institute. KRAS NM 004985.3:C.180 181de/T CinsAA. NCI Thesaurus.

Code C98431.

A complex substitution where the nucleotide sequence at positions 180 through 181 of the coding sequence of the KRAS gene has chang ed from thymine-cytosine to adenineadenine. 